Check for updates

Cite this: RSC Adv., 2018, 8, 1717

\title{
Ion specific effects on the immobilisation of charged gold nanoparticles on metal surfaces $\uparrow$
}

\author{
C. Kaulen (iD) *ab and U. Simon (iD) ab
}

Since the pioneering work of F. Hofmeister, Arch. Exp. Pathol. Pharmakol., 1888, 24, 247, ion specific effects have been steadily reported in the context of colloidal or protein stabilisation in electrolyte solutions. Although the observed effects are omnipresent in chemistry and biology, their origin is still under ferocious discussion. Here, we report on ion specific effects affecting the self-assembly of amine and carboxylic acid functionalised gold nanoparticles on metal surfaces as well as in electrolyte solution as a function of the monovalent cations $\mathrm{Li}^{+}, \mathrm{Na}^{+}, \mathrm{K}^{+}$and $\mathrm{Cs}^{+}$. Mercaptooctanoic acid and 1,8-amine-octanethiol functionalised gold nanoparticles were adsorbed on structured AuPd/Pt substrates under addition of the respective chloride salts. Furthermore, the influence of the same salts on the salt induced aggregation of these AuNP was investigated. Our results demonstrate that the assembly processes on the metal surface as well as in electrolyte solution are influenced by the addition of different cations. We attribute the observed effects to ion pairing of the functional end groups with the added cations. With these findings we introduce a new parameter to control the self-assembly of 2D AuNP arrays on solid supports or of 3D AuNP networks in solution, which could be of relevance for the fabrication of new tailor-made functional materials or for biomedical applications.

Received 18th September 2017
Accepted 19th December 2017

DOI: 10.1039/c7ra10374c

rsc.li/rsc-advances specific effects is still unclear, although several attempts at an explanation have been made. ${ }^{12,13}$

Our long term goal is to integrate ligand stabilised gold nanoparticles (AuNPs) by self-assembly on electrode surfaces, in a directed manner, thus building up hybrid nanoelectronic devices with advanced functionality. ${ }^{\mathbf{1 4}, \mathbf{1 5}}$ In this context, we presented in our previous work the directionally controlled immobilisation of tailored ligand stabilised Janus-AuNPs on gold surfaces, prefunctionalised with self-assembled monolayers (SAMs) of thiol molecules. ${ }^{16}$ Thereby, we took advantage of electrostatic and hydrophilic/hydrophobic interactions. Beyond this, we investigated the differential adsorption of ligand stabilised AuNP on pristine gold (alloyed with 30\% palladium due to the manufacturing process) and platinum surfaces depending on the $\mathrm{pH}$ and the ionic strength of the immobilisation solution. ${ }^{17}$ We found that mercaptooctanonic acid (MOA) functionalised AuNPs (Au-MOA) at pH 9 and 1,8aminooctanethiol (AOT) stabilised AuNPs (Au-AOT) at $\mathrm{pH} 3$ adsorb on platinum surfaces with high selectivity (Fig. 1b). Further, we could optimise the selectivity and the coverage density of adsorbed AuNPs by adjusting the ionic strength of the immobilisation solution. Following the theory of ion specific effects, interactions of different monovalent cations with charged $\left(-\mathrm{COO}^{-} /-\mathrm{NH}_{3}{ }^{+}\right)$and uncharged $\left(-\mathrm{COOH} /-\mathrm{NH}_{2}\right)$ end groups of the AuNPs should influence the covering density of the particles on metal surfaces as well as their salt induced precipitation (Fig. 1c). ${ }^{7,8}$ Based on these considerations, herein

\footnotetext{
aggregation of $\mathrm{Au}-\mathrm{AOT}$ with $\mathrm{LiCl}, \mathrm{NaCl}, \mathrm{KCl}, \mathrm{CsCl}$; progression of the ratio of absorbance of Au-AOT with LiCl, KCl, CsCl. See DOI: 10.1039/c7ra10374c

${ }^{a} J A R A$ - FIT, RWTH Aachen University, 52074 Aachen, Germany. E-mail: corinna

$\dagger$ Electronic supplementary informat and SEM-T images of Au-MOA, UV-Vis spectra; DLS data and SEM-T images of Au-AOT; additional SEM images of immobilised Au-MOA; additional SEM images of immobilised Au-AOT; SEM images of the aggregates of Au-MOA and Au-AOT formed during salt induced aggregation; UV-Vis spectra of salt induced aggregation of $\mathrm{Au}-\mathrm{MOA}$ with $\mathrm{LiCl}, \mathrm{NaCl}, \mathrm{KCl}, \mathrm{CsCl}$; progression of the ratio of absorbance of Au-MOA with $\mathrm{LiCl}, \mathrm{KCl}, \mathrm{CsCl}$; UV-Vis spectra of salt induced
} 
a) Kosmotropic Chaotropic

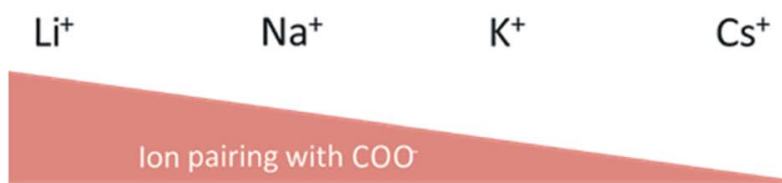

Adsorption to amine groups

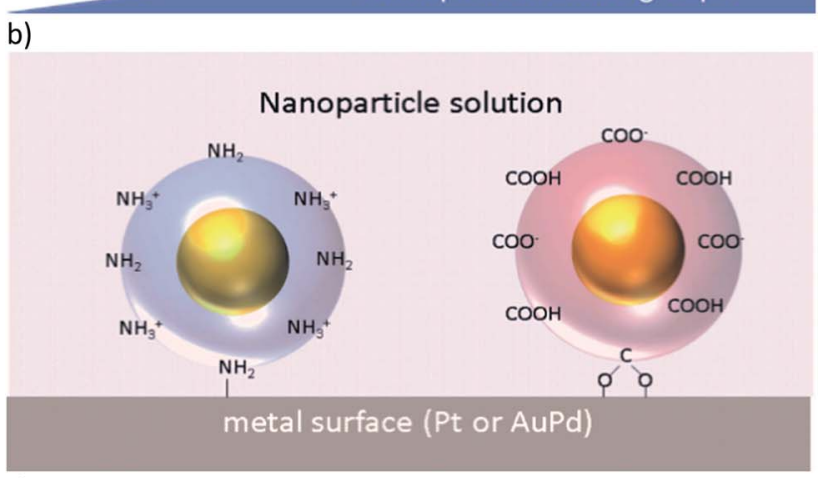

c)

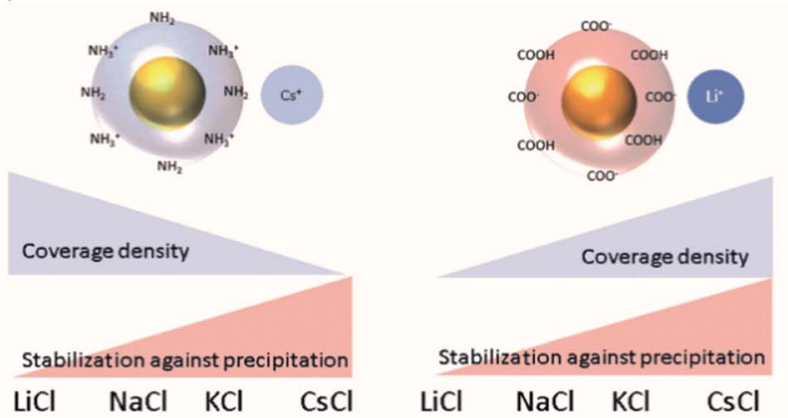

Fig. 1 (a) Kosmotropic cations form strong ion-pairs with $\mathrm{COO}^{-}$ groups, chaotropic ions adsorb to neutral $\mathrm{NH}_{2}$-groups. (b) Proposed binding model: adsorption to the metal surface by coordinative bonds of the AuNPs' end groups. (c) For Au-AOT decreasing coverage density and increasing stability against precipitation is expected for more chaotropic cations, while for Au-MOA an inverse trend in coverage density and similar stability against precipitation is expected.

we present a detailed study on the adsorption characteristics of amine terminated Au-AOT and carboxylic acid terminated $\mathrm{Au}-$ MOA on pristine AuPd and Pt surfaces under the influence of different monovalent chloride salts $\mathrm{MCl}\left(\mathrm{M}=\mathrm{Li}^{+}, \mathrm{Na}^{+}, \mathrm{K}^{+}, \mathrm{Cs}^{+}\right)$. We chose AuPd and Pt as metal surfaces to determine the binding affinity of amine and carboxylic acid groups to these technically relevant electrode materials. Furthermore, we evaluate the colloidal stabilities of the Au-MOA and Au-AOT solutions with addition of the respective salts MCl by UV-Vis spectroscopy and we analyse the formed aggregates by SEM. Finally, the results of these investigations could be applied usefully on the self-assembly of AuNP for the construction of metamaterials,$^{18}$ sensing of biomolecules ${ }^{19,20}$ or the formation of tailor-made AuNP aggregates for theranostics. ${ }^{21}$

\section{Experimental}

\section{Materials}

The following chemicals were purchased from Sigma-Aldrich Chemie $\mathrm{GmbH}$ and were used as received: hydrogen tetrachloroaurate(III) trihydrate $\left(\mathrm{HAuCl}_{4} \cdot 3 \mathrm{H}_{2} \mathrm{O}\right)$, trisodium citrate dihydrate $\left(\mathrm{C}_{6} \mathrm{H}_{5} \mathrm{Na}_{3} \mathrm{O}_{7} \cdot 2 \mathrm{H}_{2} \mathrm{O}\right), \quad$ MOA, 4-(2-hydroxyethyl)-1piperazine-ethanesulfonic acid (HEPES), tris(hydroxymethyl) aminomethane (TRIS), and 3-triethoxysilylpropylamine (APTES). Glass beads were purchased from Carl Roth GmbH + Co. KG. AOT was purchased from Dojindo Molecular Technologies, Inc. All glassware was cleaned with aqua regia and rinsed with copious amount of water prior to use. Ultrapure water with a conductivity $<55 \mathrm{nS} \mathrm{cm}^{-1}$ was used for all procedures.

\section{Instrumentation and particle characterisation}

UV-Vis absorption spectra were recorded with a JASCO V-630 spectrophotometer, dynamic light scattering (DLS) measurements were performed with a Malvern Zetasizer Nano S, He-Nelaser $\lambda=633 \mathrm{~nm}, P=4 \mathrm{~mW}, \theta=173^{\circ}$ in order to determine the hydrodynamic radii of single AuNP and AuNP agglomerates, respectively, Scanning Electron Microscopy (SEM) was conducted with a high resolution Field Emission Scanning Electron Microscope (FE-SEM, LEO/ZEISS Supra 35 VP, Oberkochen, Germany) and, particle concentrations were estimated from gold concentrations determined by atomic absorption spectroscopy (AAS, Shimadzu AA-7000, Tokyo, Japan).

\section{Substrate fabrication}

Structured Pt/AuPd substrates were fabricated as previously described. ${ }^{17}$ Briefly, a Si (100) substrate $\left(1 \times 1\right.$ inch, $\left.1-5 \Omega \mathrm{cm}^{-1}\right)$ was covered with an insulating $\mathrm{SiO}_{2}$ layer $(430 \mathrm{~nm})$ which was created by oxidation of $\mathrm{Si}$ using thermal dissociation of water vapour at $1500{ }^{\circ} \mathrm{C}$ for $50 \mathrm{~min}$. Subsequently, a full plain metallic layer was fabricated by vapour deposition of titanium (adhesion layer, $10-20 \mathrm{~nm})$ and platinum $(100 \mathrm{~nm}$, deposition rate $2 \mathrm{~nm} \mathrm{~s}^{-1}$, pressure $<1 \times 10^{-7}$ mbar) onto the $\mathrm{Si} / \mathrm{SiO}_{2}$ substrate. Thin AuPd (30\% Pd) lines with a thickness of $15 \mathrm{~nm}$ and with varying widths between $500 \mathrm{~nm}$ and $1 \mu \mathrm{m}$ were defined via e-beam lithography in a lift-off process on top of the platinum layer. After lift-off the sample was rinsed with 2-propanol and then dried in a stream of $\mathrm{N}_{2}$.

\section{Adsorption studies}

The Pt/AuPd substrates were cut into $5 \times 5 \mathrm{~mm}^{2}$ pieces. The substrate pieces were washed with $5 \mathrm{~mL}$ ethanol and subsequently treated with oxygen plasma $\left(0.4 \% \mathrm{O}_{2}, 100 \mathrm{~W}\right)$ for 2 minutes in order to remove organic residues. $10 \mu \mathrm{L}$ of the AuMOA solution in HEPES/TRIS buffer $(10 \mathrm{mM})$ at $\mathrm{pH} 9$ (particle concentration of $9.6 \times 10^{-9} \mathrm{~mol} \mathrm{~L}^{-1}$ ) or the Au-AOT in diluted $\mathrm{HCl}$ at $\mathrm{pH} 3$ (particle concentration of $4.4 \times 10^{-9} \mathrm{~mol} \mathrm{~L}^{-1}$ ) was drop cast onto the cleaned surface, immediately after plasma treatment. The sample was kept in a $\mathrm{H}_{2} \mathrm{O}$ saturated atmosphere to prevent evaporation of solvent. After $20 \mathrm{~min}$., the substrate was rinsed with water in order to remove unbound AuNPs and 
dried in a nitrogen stream. The substrates were analysed by SEM and statistical evaluations of the particle density were performed related to an area of $1 \mu \mathrm{m}^{2}$ on 9 different positions of the substrate.

\section{Colloidal stability studies}

Au-MOA and Au-AOT were synthesized and purified as previously described. ${ }^{\mathbf{1 4}}$ After the last centrifugation step Au-MOA were redispersed in $10 \mathrm{mM}$ HEPES/TRIS buffer at $\mathrm{pH} 9$ and the required amount of a $1 \mathrm{M} \mathrm{MCl}$ solution was added to final salt concentrations between $40 \mathrm{mM}$ and $120 \mathrm{mM}$. The Au-MOA concentration was $4.5 \times 10^{-9} \mathrm{~mol} \mathrm{~L}^{-1}$. Au-AOT were redispersed in $10^{-3} \mathrm{M} \mathrm{HCl}(\mathrm{pH} 3)$ and the required amounts of $1 \mathrm{M}$ $\mathrm{MCl}$ solutions were added to final salt concentrations of $100 \mathrm{mM}$ to $250 \mathrm{mM}$. The Au-AOT concentration was $2.2 \times$ $10^{-9} \mathrm{~mol} \mathrm{~L}^{-1}$. Immediately after addition of the salt solution UV-Vis spectra of the colloid solutions were collected within 3 minute intervals for one hour.

\section{SEM investigation of the formed aggregates}

A Au-MOA solution $\left(4.5 \times 10^{-9} \mathrm{~mol} \mathrm{~L}^{-1}\right)$ in HEPES/TRIS at pH 9 with $45 \mathrm{mmol} \mathrm{NaCl}$ and a Au-AOT solution $\left(2.2 \times 10^{-9} \mathrm{~mol} \mathrm{~L}^{-1}\right)$ in $10^{-3} \mathrm{M} \mathrm{HCl}(\mathrm{pH} \mathrm{3})$ with $125 \mathrm{mmol} \mathrm{NaCl}$ were prepared. Immediately after mixing $10 \mu \mathrm{L}$ samples were taken every five minutes and deposited on a carbon-coated TEM grid. After another five minutes, the solution was removed with a KIM wipe and the grids were allowed to dry at $20 \%$ air humidity and $20^{\circ} \mathrm{C}$. SEM images were acquired in transmission mode.

\section{Results and discussion}

\section{Adsorption of Au-MOA and Au-AOT on Pt and AuPd surfaces in dependence of the addition of different monovalent chloride salts}

Au-MOA nanoparticles with a mean diameter of $13.1 \mathrm{~nm} \pm$ $1.3 \mathrm{~nm}$ were synthesized from citrate stabilised AuNPs by a ligand exchange reaction with MOA as previously published, ${ }^{17}$ purified by centrifugation and redispersed in HEPES/TRIS buffer at $\mathrm{pH}$ 9. For the synthesis of Au-AOT (mean diameter $14.9 \mathrm{~nm} \pm 1.2 \mathrm{~nm}$ ) a solid phase supported approach was applied in order to prevent irreversible aggregation of the citrate stabilised AuNPs during ligand exchange with AOT. ${ }^{17}$ After synthesis the Au-AOT were purified by centrifugation and redispersed in diluted $\mathrm{HCl}$ solution at $\mathrm{pH}$ 3. The corresponding UV-Vis spectra, the hydrodynamic diameter and zeta-potentials determined by DLS, and representative SEM images are displayed in the ESI (Fig. S1-S4 in the ESI $\dagger$ ). A zeta-potential change from $-69.8 \pm 7.9 \mathrm{mV}$ for citrate stabilised AuNP to $-42.5 \pm 9.8 \mathrm{mV}$ at $\mathrm{pH} 9$ for $\mathrm{Au}-\mathrm{MOA}$ and to $+60.7 \pm 8.5 \mathrm{mV}$ for $\mathrm{Au}-\mathrm{AOT}$ at $\mathrm{pH} 3$ clearly indicates successful ligand exchange. In a first set of experiments, we investigated the effect of the monovalent cations $\mathrm{M}=\mathrm{Li}^{+}, \mathrm{Na}^{+}, \mathrm{K}^{+}$, and $\mathrm{Cs}^{+}$on the adsorption characteristics of carboxylic acid terminated AuNPs (Au-MOA) on AuPd and Pt surfaces. We chose this row of monovalent cations, because the investigated Au-AOT and Au-MOA are stable at moderately enhanced electrolyte concentrations of these salts, while already very low concentrations of divalent salts like $\mathrm{Mg}^{2+}$ or $\mathrm{Ca}^{2+}$ lead to aggregation of Au-MOA. ${ }^{22}$ Therefore, neither, the influence of divalent cations, nor the impact of different monovalent anions was investigated, as $\mathrm{Au}$ AOT showed oxidative degradation of the Au core upon addition of $\mathrm{I}^{-}$or $\mathrm{Br}^{-} .{ }^{23}$ In order to transfer our results to experiments with nanoelectrode structures (nanogaps), we chose specially designed substrates, consisting of a layer of Pt (100 nm) with 1 $\mu \mathrm{m}$ broad AuPd (30\% Pd, $15 \mathrm{~nm}$ ) lines on it to enable a directed deposition of the functionalised AuNP via self-assembly. ${ }^{17}$ This design of the structured substrates ensures that the adsorption of the AuNPs on the AuPd and Pt surfaces takes place under identical conditions regarding temperature, $\mathrm{pH}$ and AuNP concentration. Samples were prepared by adding an aqueous solution of the respective $\mathrm{MCl}$ solution $\left(\mathrm{M}=\mathrm{Li}^{+}, \mathrm{Na}^{+}, \mathrm{K}^{+}\right.$, and $\left.\mathrm{Cs}^{+}\right)$to each Au-MOA-HEPES/TRIS dispersion, whereby the added $\mathrm{MCl}$ amount was adjusted according to a final salt concentration of $10 \mathrm{mM}$. Additionally, a reference sample in pure HEPES/TRIS buffer was set up. $10 \mu \mathrm{L}$ of the freshly prepared Au-MOA solutions were drop cast on the structured AuPd/Pt substrates. This kind of sample preparation was used to emulate the trapping of the AuNPs between nanostructured electrodes. During incubation the substrates were kept in a water saturated atmosphere and were gently swayed in order to avoid the so called "coffee ring effect". ${ }^{24}$ After $45 \mathrm{~min}$, the substrates were rinsed with water and dried in a nitrogen stream. SEM images were taken from nine equally distributed different spots on each substrate. Firstly, on the surface of the reference sample without any salt addition we could detect only single adsorbed particles (Fig. 2a). Which illustrates that salt addition is necessary to assist the adsorption process. Secondly, all images of the samples with addition of the different salts show moderate particle coverage on platinum but no adsorbed Au-MOA on the AuPd surface. The Au-MOA are present as single particles or small assemblies of 2-10 particles. The density of adsorbed AuNP ( $\Gamma$ Pt and $\Gamma$ AuPd) was determined by counting the adsorbed AuNP within in each spot comprising an area of $1 \mu^{2}$. Exemplary SEM images as well as respective particle surface covering densities are shown in Fig. 3 (see Fig. S5 in the ESI $\dagger$ for additional images).

These results reveal that the highest surface coverage density was determined in the presence of $\mathrm{Li}^{+}$and the lowest in the presence of $\mathrm{Na}^{+}$. In the presence of $\mathrm{K}^{+}$and $\mathrm{Cs}^{+}$the amount of AuNP was in between the aforementioned. A certain variation in covering density was observed, which we assign to the concentration fluctuation of the AuNP solution during sample preparation. Nevertheless, the fluctuation does not affect the determined trend. Moreover, in all experiments we found preferential adsorption on the Pt surface, which portends that the mechanism of adsorption changes not substantially with addition of the different cations.

In a second set of experiments we investigated the adsorption of Au-AOT in the presence of the same row of chloride salts $\mathrm{MCl}(\mathrm{M}=\mathrm{Li}, \mathrm{Na}, \mathrm{K}, \mathrm{Cs})$. An aqueous solution of the respective $\mathrm{MCl}$ solution was added to each Au-AOT dispersion, whereby the added $\mathrm{MCl}$ amount was adjusted accordingly to a final salt concentration of $10 \mathrm{mM}$. Here, we also provided a reference 
a)

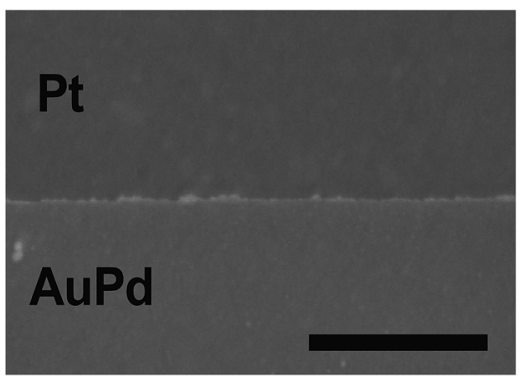

b)

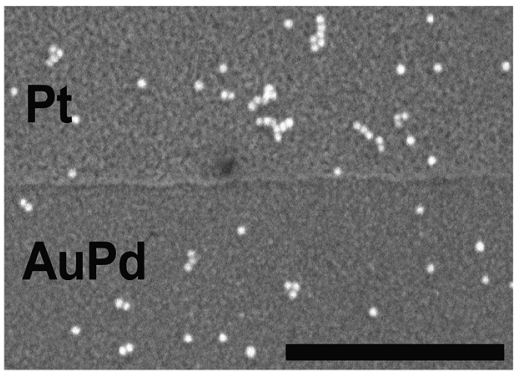

c)

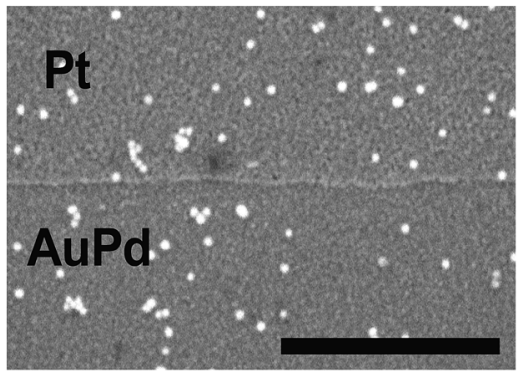

Fig. 2 SEM images of adsorption experiments on substrates consisting of a $100 \mathrm{~nm}$ Pt layer with $15 \mathrm{~nm}$ AuPd structures on it without salt addition, (a) Au-MOA in HEPES/TRIS at pH 9; (b) Au-AOT in diluted acetic acid at $\mathrm{pH}$ 3; (c) Au-AOT in diluted $\mathrm{HCl}$ at $\mathrm{pH}$ 3. Scale bar represents $500 \mathrm{~nm}$.

sample without $\mathrm{MCl}$ addition. Beyond this, we prepared a chloride free Au-AOT solution by redispersing the Au-AOT in diluted acetic acid, to understand the influence of the chloride on the adsorption process. $10 \mu \mathrm{L}$ of the freshly prepared Au-AOT solutions were drop cast on the structured AuPd/Pt substrates and the substrates were prepared for SEM analysis in the same way as for the Au-MOA. The SEM images of the reference samples are displayed in Fig. $2 \mathrm{~b}$ and c. On both reference samples Au-AOT are adsorbed with low covering densities on either Pt or AuPd surfaces with no specific preference. Fig. 4 shows the respective SEM images of the adsorption experiments of Au-AOT with added $\mathrm{MCl}$ as well as the evaluated coverage densities (see Fig. S6 in the ESI† for further images). Here a submonolayer of particles had formed on the Pt surface while several single Au-AOT were present on the AuPd surface. We evaluated the covering densities for amine terminated AuNP on Pt and AuPd as described for Au-MOA. This evaluation revealed decreasing AuNP density on Pt in the following order: $\mathrm{Li}>\mathrm{Na} \approx$ $\mathrm{K}>\mathrm{Cs}$. Overall, the Au-AOT surface coverage densities on AuPd were significantly lower than on $\mathrm{Pt}$, thus pointing out that there is no influence of the different cations on the selectivity of the adsorption. However, our results reveal that there is indeed an influence of the different cations on the amount of adsorbed AuNPs. In order to explain the observed effect, we took into account (i) the influences of salt ions on the AuNPs' end-groups (Fig. 1c) and (ii) the proposed binding model of the AuNP on the metal surface (Fig. 1b).

Concerning (i), we expected ion pairing of kosmotropic cations with the charged carboxylate groups of Au-MOA and charge compensation by adsorption of cations to neutral carboxylic acid groups. The same holds for the amine end groups of Au-AOT, hence there will be ion pairing of chloride anions with positively charged ammonium groups as well as adsorption of the different cations to the neutral amine groups in various extent at the AuNPs' end groups. The number of charged end groups on AuNP stabilized by a SAM with $\mathrm{pH}$ sensitive groups like carboxylic acid or amine groups, depends on the $\mathrm{pH}$ of the solution. As pointed out by Grzybowski et al., the $\mathrm{p} K_{\mathrm{a}}$ of carboxylic acid groups rises significantly when the groups are densely packed within a SAM on a gold surface. ${ }^{25}$ a)

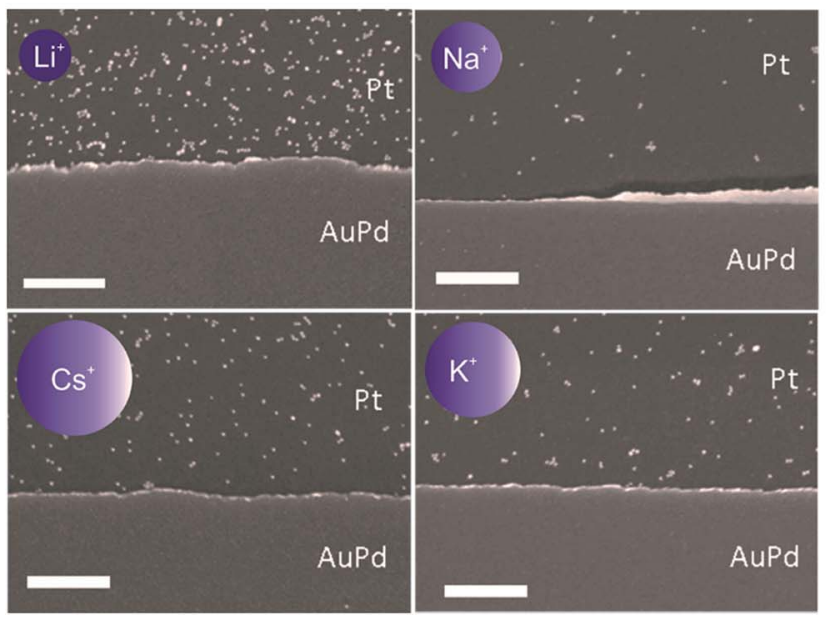

b)

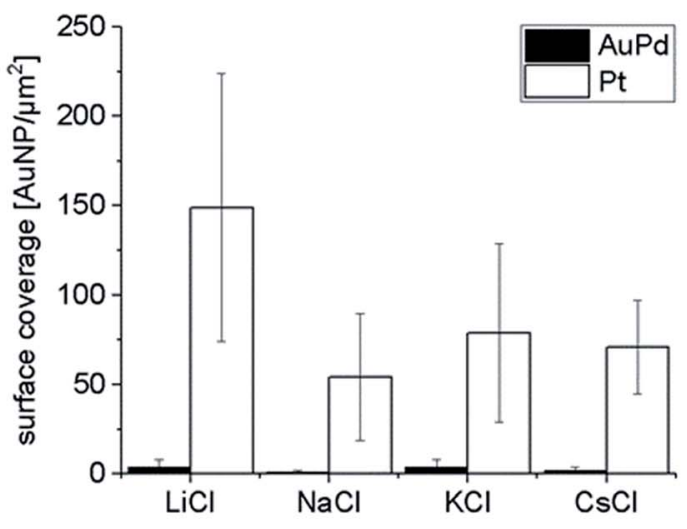

Fig. 3 (a) Representative SEM images of adsorption experiments of $A u-M O A$ with added monovalent salts $\mathrm{MCl}(\mathrm{M}=\mathrm{Li}, \mathrm{Na}$, K, $\mathrm{Cs}$, the size of the schematic ions correlates to the sizes of the corresponding ionic radii) and (b) the determined covering densities, where the error bars show the mean of three experiments and the nine different spots which were analysed on each substrate, scale bar represents $500 \mathrm{~nm}$. 
a)

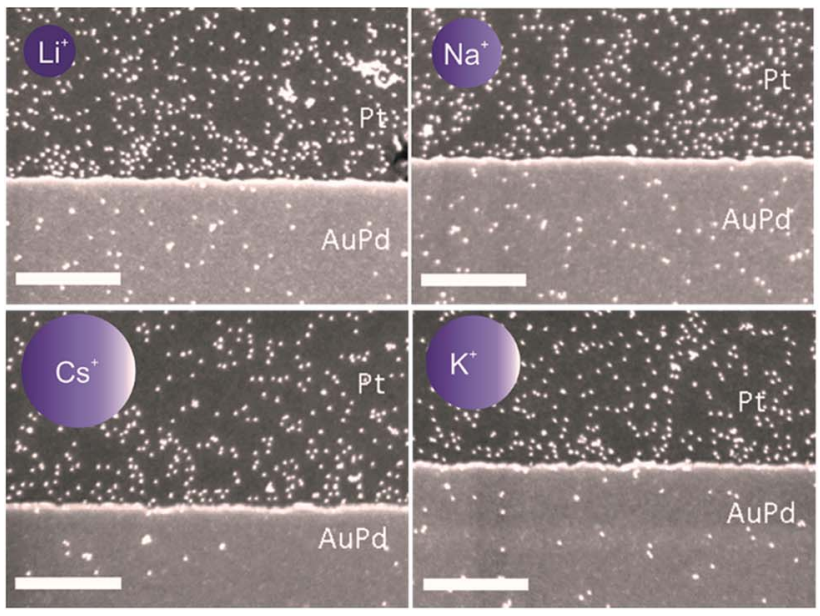

b)

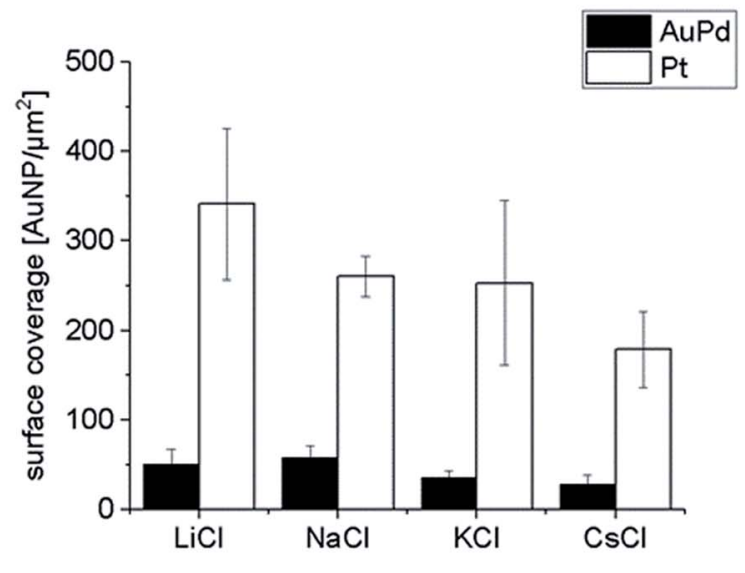

Fig. 4 (a) Representative SEM images of adsorption experiments of Au-AOT with added monovalent salts $\mathrm{MCl}(\mathrm{M}=\mathrm{Li}$, $\mathrm{Na}$, $\mathrm{K}, \mathrm{Cs}$, the size of the schematic ions correlates to the sizes of the corresponding ionic radii) and (b) the determined covering densities, where the error bars show the mean of three experiments and the nine different spots which were analysed on each substrate, scale bar represents $500 \mathrm{~nm}$.

Thus, the $\mathrm{p} K_{\mathrm{a}}$ of the carboxylic acid groups on Au-MOA lies between the $\mathrm{p} K_{\mathrm{a}}$ of free MOA molecules in solution $\left(\mathrm{p} K_{\mathrm{a}} \sim 4.8\right)$ and the $\mathrm{p} K_{\mathrm{a}}$ of MOA within a monolayer on a flat gold surface $\left(\mathrm{p} K_{\mathrm{a}} \sim 10\right) .{ }^{26}$ That implies that at $\mathrm{pH} 9$ the ligand shell of the AuMOA exists of neutral carboxylic acid end groups and of negatively charged carboxylate end groups. The same applies to the amine end groups of Au-AOT at $\mathrm{pH} 3$ hence, there will be neutral amine groups as well as positively charged ammonium groups on the AuNP surface (Fig. 1b).

Concerning (ii), if adsorption of AuNPs occurs via coordinative binding of the terminal end groups and the metal surface, we expect highest covering densities, when many terminal groups with free electron pairs are available. i.e. when no ion pairing of the $-\mathrm{COO}^{-}$and the $-\mathrm{NH}_{2}$ groups with ions takes place.

To understand the interactions of the charged end groups with ions we investigated the aggregation behaviour of the Au-AOT and $\mathrm{Au}-\mathrm{MOA}$ in the respective electrolyte solutions. Salt induced aggregation occurs by depletion of the electrochemical double layer surrounding the particles in aqueous solution. ${ }^{27}$ Aggregation starts when the charges of the end groups are shielded due to the formation of ion-pairs with the available ions in solution and thereby the electrostatic repulsion minimizes, so that particle collisions lead to precipitation. This should lead to stabilisation of $\mathrm{Au}-\mathrm{MOA}$ and Au-AOT in electrolyte solutions with chaotrope cations (Fig. 1c). Therefore, we performed salt induced aggregation experiments in order to evaluate the interactions of the respective terminal group $\left(-\mathrm{COOH}\right.$ or $\left.-\mathrm{NH}_{2}\right)$ with the particular $\mathrm{M}^{+}$cations $\left(\mathrm{M}^{+}=\mathrm{Li}^{+}, \mathrm{Na}^{+}, \mathrm{K}^{+}, \mathrm{Cs}^{+}\right)$.

\section{Time dependent aggregation of Au-MOA and Au-AOT upon addition of different salts $\mathrm{MCl}(\mathrm{M}=\mathrm{Li}, \mathrm{Na}, \mathrm{K}, \mathrm{Cs})$}

A stock solution of Au-MOA in HEPES/TRIS buffer at pH 9 was prepared. Samples were taken from these stock solution and different amounts of a $1 \mathrm{M} \mathrm{MCl}$ solution were added leading to final salt concentrations from $40 \mathrm{mM}$ to $250 \mathrm{mM}$. Immediately after mixing of the sample, aggregation was observed by UV-Vis spectroscopy. Additionally, we took for one representative experiment, namely the aggregation with $45 \mathrm{mmol} \mathrm{NaCl}$, every five minutes samples and deposited them on TEM grids in order to determine the shape and size of the formed aggregates. The SEM images illustrate quite vivid that within five minutes aggregates with a size of up to $100 \mathrm{~nm}$ are formed and after 15 minutes larger up to $500 \mathrm{~nm}$ long and $100 \mathrm{~nm}$ broad, most elongated aggregates dominate (see Table 1 in the ESI $\dagger$ ). These aggregates lead to a significant red shift of the AuNPs' plasmon resonance. While single AuNPs show a maximum plasmon resonance at $520 \mathrm{~nm}$, aggregates absorb at higher wavelengths. Therefore, to determine the ratio of single particles to aggregates the ratio $R$ of absorbance at $520 \mathrm{~nm}$ to the absorbance at $620 \mathrm{~nm}$ was calculated and plotted as a function of time..$^{23,27-29}$ The UV-Vis spectra of the aggregation experiment of Au-MOA with $\mathrm{NaCl}$ and the derived time-dependent course of the ratio $R$ of absorbance are depicted in Fig. $5 \mathrm{a}$ and b. All UV-Vis spectra and the aggregation curves of Au-MOA with $\mathrm{LiCl}, \mathrm{NaCl}, \mathrm{KCl}$, and $\mathrm{CsCl}$ are given in the ESI (Fig. S7-S31†). In order to compare the stability of the AuNPs in the different salt solutions, the aggregation curves were fitted by a first order exponential decay function (eqn (1)). From this fit curves the rate constants of the aggregation were calculated by $k=t^{-1}$.

$$
y=y_{0}+A_{1} e^{\frac{-x}{t}}
$$

The derived $k$ values are plotted as a function of electrolyte concentration (Fig. 5c), where values close to zero reflect slow aggregation, while values close to 1 and higher express fast aggregation. Based on these results the stability of Au-MOA is highest in $\mathrm{CsCl}$, reduced in $\mathrm{LiCl}$ and lowest in $\mathrm{KCl}$ and $\mathrm{NaCl}$. That means, the terminal carboxylate group forms strong ion pairs with $\mathrm{Na}^{+}$and $\mathrm{K}^{+}$, while solvent separated ion pairs are 


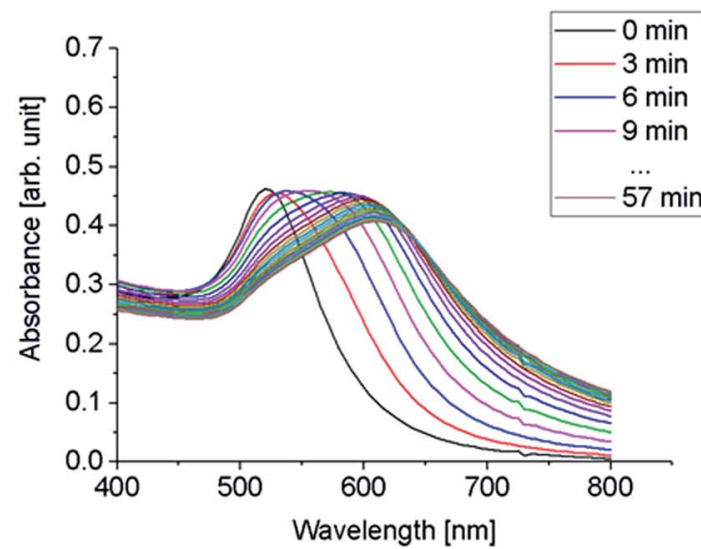

a)

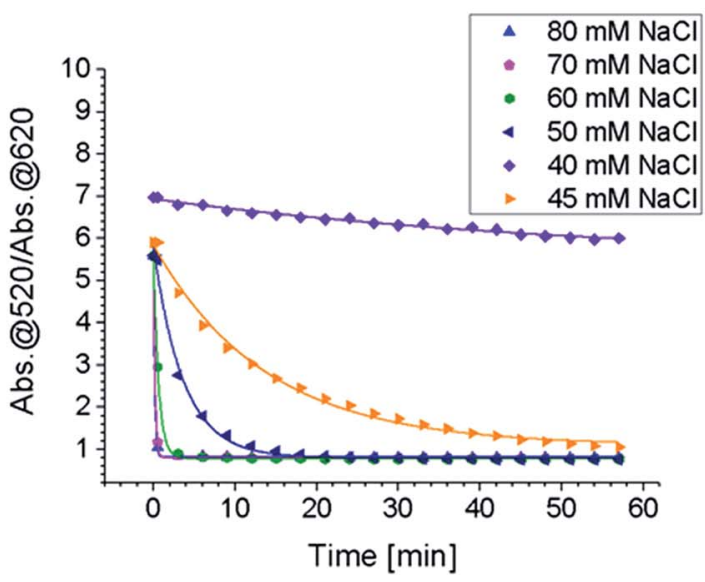

b)

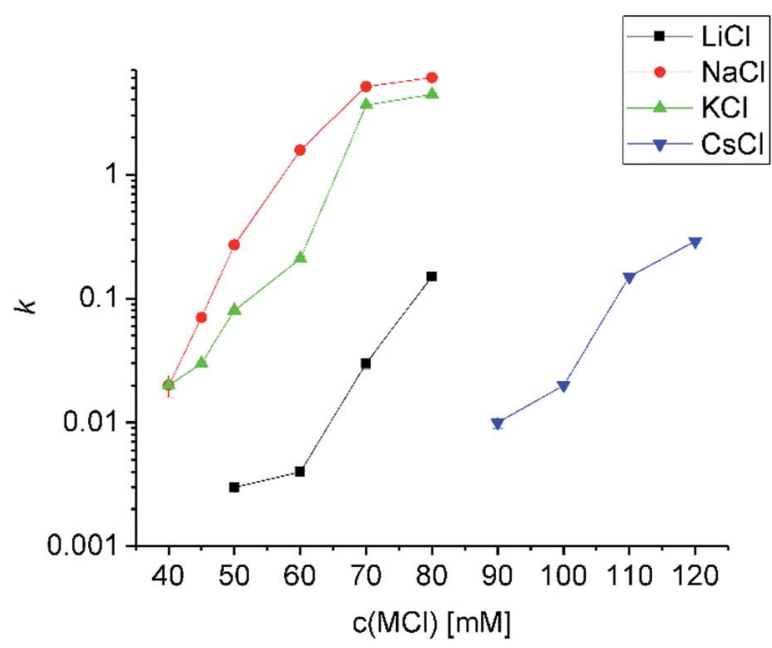

c)

Fig. 5 (a) Time dependent absorption spectra of Au-MOA in HEPES/ TRIS at pH 9 after adding $\mathrm{NaCl}$ (final concentration $50 \mathrm{mM}$ ), spectra were taken every 3 minutes over $1 \mathrm{~h}$. (b) Progression of the ratio of absorbance $R$ (symbols) for $\mathrm{NaCl}$ concentrations from $40 \mathrm{mM}$ to $80 \mathrm{mM}$ and the respective fit curves (lines) fitted by a first order exponential decay function. (c) Semi-logarithmic plot of the aggregation constant $k$ as a function of electrolyte concentration for the time dependent aggregation of Au-MOA. The higher the value of $k$, the faster the aggregation proceeds. formed with $\mathrm{Li}^{+}$and $\mathrm{Cs}^{+}$. According to the theory of ion specific effects, the carboxylic acid group is considered as a hard anion, which is expected to form strong ion pairs with hard cations like $\mathrm{Li}^{+}$and $\mathrm{Na}^{+}$. This correlates with our observations for $\mathrm{Na}^{+}$but $\mathrm{Li}^{+}$seems to act more like a soft cation. One explanation for this phenomenon can be derived from the fact that the aggregation of the Au-MOA also occurs by charge compensation due to adsorption of cations to the neutral carboxylic acid groups: the adsorption of $\mathrm{Li}^{+}$at the neutral $\mathrm{COOH}$ groups is hindered by the strong hydration shell of $\mathrm{Li}^{+}$, thus preventing charge compensation of the overall negatively charged Au-MOA and thereby hampering salt induced aggregation, as shown also on $\mathrm{COOH}$ terminated SAMs by MD simulations. ${ }^{30,31}$ Further, a comparable exception is reported for ion pairing of monovalent salts with carboxylate groups of acetate and glycine. ${ }^{32}$

Aggregation of Au-AOT was investigated in the same manner as described for Au-MOA. For these positively charged AuNP we expect a less pronounced influence of the different cations, because ion pairing of the cationic $-\mathrm{NH}_{3}{ }^{+}$end group with chloride anions takes place in the same degree for all investigated salts. Nevertheless, we expected a certain effect due to adsorption of cations to the neutral $\mathrm{NH}_{2}$-groups, which results in an increase of positive charge and therefore in a higher stability in highly concentrated salt solutions. The SEM images of the Au-AOT samples, which were prepared during the aggregation process, show fast formation of rather ellipsoidal aggregates with a size of $c a$. $50 \mathrm{~nm}$ after five minutes and of larger sized $(200-300 \mathrm{~nm}$ ) assemblies after 15 minutes (see Table S1 in the ESI $\dagger$ ). In the UV-Vis, differing from the aggregation behaviour of Au-MOA, where the aggregation leads to formation of a new absorption maximum at higher wavelength, the respective spectra of the time dependent measurements for Au-AOT show an increase of the absorption within the wavelength range from $600 \mathrm{~nm}$ to $800 \mathrm{~nm}$, but no distinct new maximum (Fig. 6a). Therefore, the ratio $R$ of absorbance was determined as the ratio of the absorption at $520 \mathrm{~nm}$ to the absorption at $700 \mathrm{~nm}$ (Fig. 6b). Fitting of the time dependent ratio $R$ of absorbance retrieved the aggregation constant $k$ for the aggregation of Au-AOT with $\mathrm{MCl}(\mathrm{Li}, \mathrm{Na}, \mathrm{K}, \mathrm{Cs})$ at salt concentrations from 100-275 mM (see Fig. S32-S56 in the ESI $\dagger$ for all corresponding UV-Vis spectra).

The evaluation of the $k$ values shows that aggregation occurs at low salt concentrations for $\mathrm{LiCl}$ and $\mathrm{NaCl}$ while, in the case of $\mathrm{KCl}$ aggregation starts at higher salt concentration, and we observed highest stability with $\mathrm{CsCl}$ (Fig. 6c). These observations are in good accordance with the trend expected from literature. ${ }^{2,7,12}$ The kosmotropic cations $\mathrm{Li}^{+}$and $\mathrm{Na}^{+}$do not adsorb to the neutral chaotropic amine groups of Au-AOT, therefore no additional stabilisation due to added positive charges is possible. Accordingly, aggregation is observed at low salt concentrations with kosmotropic cations, e.g. at $125 \mathrm{mM}$ with LiCl. The chaotropic cations $\mathrm{K}^{+}$and $\mathrm{Cs}^{+}$adsorb to the amine end groups and thereby enhance the positive charge of the Au-AOT which results in a higher stability of these AuNP against salt induced aggregation, e.g. Au-AOT are still stable in $200 \mathrm{mM} \mathrm{CsCl}$ as electrolyte. Hence, the aggregation behavior of 


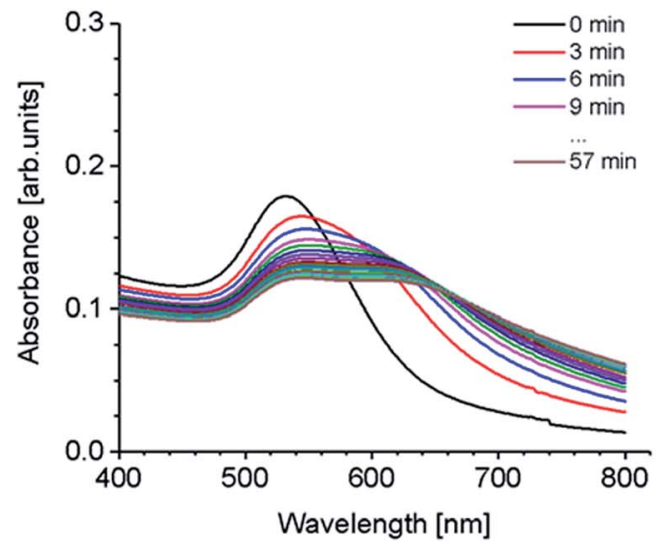

a)

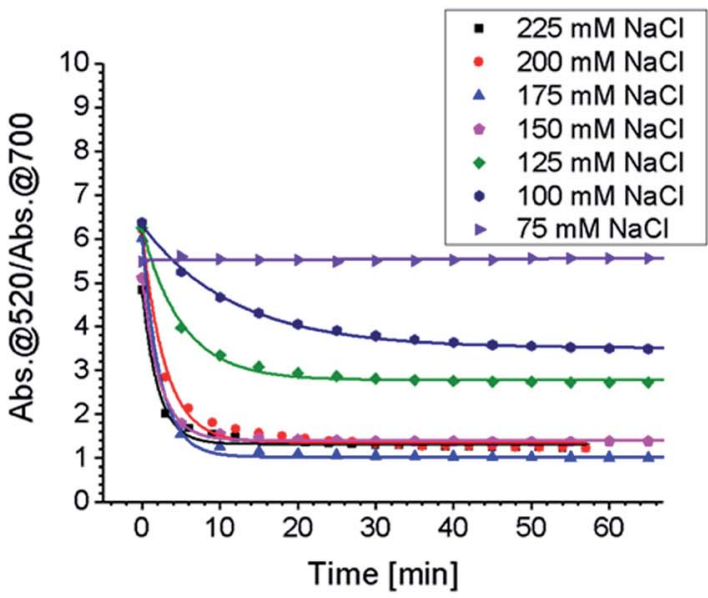

b)

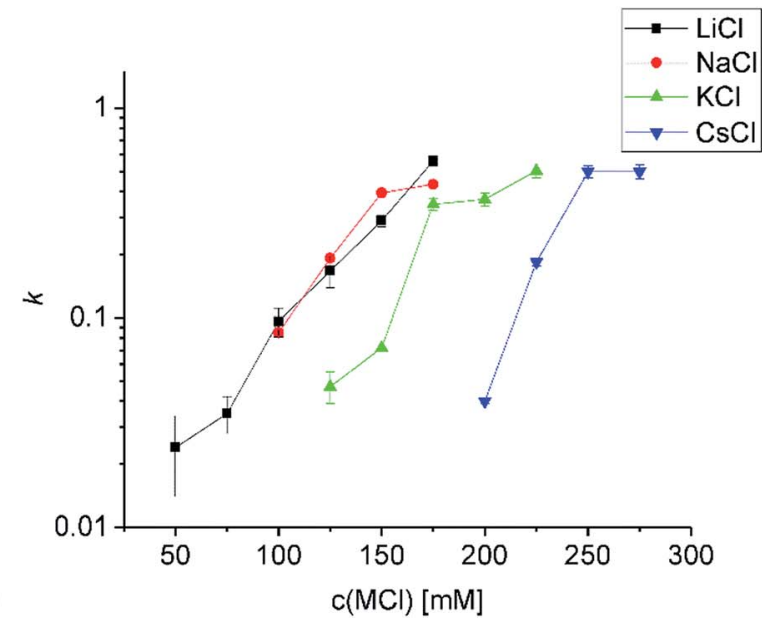

Fig. 6 (a) Time dependent absorption spectra of $\mathrm{Au}-\mathrm{AOT}$ in $\mathrm{H}_{2} \mathrm{O} / \mathrm{HCl}$ at $\mathrm{pH} 3$ after adding $\mathrm{NaCl}$ (final concentration $200 \mathrm{mM}$ ), spectra were taken every 3 minutes over $1 \mathrm{~h}$. (b) Time course of the ratio $R$ of absorbance for $\mathrm{NaCl}$ concentrations from 75 to $225 \mathrm{mM}$ (symbols) and the respective fit curves (lines) fitted by a first order exponential decay function. (c) Semi-logarithmic plot of $k$ as a function of electrolyte concentration for the time dependent aggregation of Au-AOT. The higher the value $k$, the faster the aggregation proceeds.
$\mathrm{Au}-\mathrm{AOT}$ in different monovalent chloride salts follows the trend expected from the theory of ion specific effects., ${ }^{2,712}$

How do these results correlate to the adsorption of the investigated AuNP on AuPd or Pt metal surfaces? We proposed that the binding of the AuNP is achieved upon coordinative binding of available electron pairs of the functional end groups $-\mathrm{COO}^{-}$and $-\mathrm{NH}_{2}$ (Fig. 1b). Therefore, in case of Au-AOT, the covering density should decrease with increasing adsorption of cations to the $\mathrm{NH}_{2}$-groups, i.e. with increasing ion radius of $\mathrm{M}^{+}$. From the aggregation experiments with Au-MOA we can conclude that the covering density should increase with increasing ion radius, with an exception for $\mathrm{Li}^{+}$. A comparison with the experimentally obtained covering densities (see Fig. 3 and 4) confirms these expectations, which proves the proposed coordinative binding mechanism through the AuNPs' terminal groups.

\section{Conclusions}

This experimental study gives evidence of ion specific effects on the adsorption of thiol stabilised AuNP with carboxylic acid (Au$\mathrm{MOA}$ ) and amine (Au-AOT) terminal groups, respectively, on platinum and gold/palladium surfaces. We investigated the covering density of Au-MOA and Au-AOT on platinum and gold/palladium as well as their salt induced precipitation as function of different monovalent salt solutions $\left(\mathrm{MCl}, \mathrm{M}^{+}=\mathrm{Li}^{+}, \mathrm{Na}^{+}, \mathrm{K}^{+}, \mathrm{Cs}^{+}\right)$. We found for Au-MOA the highest covering density upon addition of $\mathrm{Li}^{+}$, a minimum with $\mathrm{Na}^{+}$and in between for $\mathrm{K}^{+}$and $\mathrm{Cs}^{+}$. The aggregation experiments led to comparable trends, i.e., for Au-MOA fast aggregation with $\mathrm{Na}^{+}$and $\mathrm{K}^{+}$, delayed aggregation with $\mathrm{Li}^{+}$and longest stability in $\mathrm{Cs}^{+}$. In the adsorption experiments with $\mathrm{Au}-\mathrm{AOT}$ a maximum covering density was observed with $\mathrm{Li}^{+}$, which decreased with decreasing ion radius of the added cation. The salt induced aggregation of Au-AOT exhibited increasing stability in the order $\mathrm{LiCl}<\mathrm{NaCl}<\mathrm{KCl}<\mathrm{CsCl}$. The observed trends point to an increased stabilisation in solution with increasing ion radius of the added cations due to their adsorption to neutral amine groups. Based on a proposed binding of the AuNPs via donation of free electron pairs of the terminal groups to the metal surface, the results of the aggregation experiments fit well to the results of the adsorption experiments, which confirms our binding model.

To sum up, we could elucidate the interactions of the terminal groups $-\mathrm{COO}^{-}$and $-\mathrm{NH}_{2}$ with different monovalent cations by combination of aggregation and adsorption experiments. These results allow further control of the self-assembly of charged NPs in solution and on metal surfaces with respect to covering density and 3D network formation, which can be utilized for the development of new sensors, ${ }^{33}$ metamaterials, ${ }^{18}$ and the implementation of functionalised AuNPs within nanoelectronic devices. ${ }^{34}$

\section{Conflicts of interest}

There are no conflicts to declare.

\section{Acknowledgements}

We thank Jutta Kiesgen and Birgit Hahn for taking the SEM images and Rebecca Liffmann for performing the SEM 
investigation of the salt induced aggregation and the reference experiments of the adsorption of Au-AOT without MCl. Further, we thank Toshimitso Sato, Simon Clauser, and Shannon Potts for performing the aggregation experiments and evaluating AuNP covering densities. The authors thank Dr Silvia Karthäuser for providing the Pt/AuPd substrates as well as Dr Melanie Homberger and Dr Michael Noyong for helpful discussions on the manuscript. This work was financially supported by the German Research Foundation (DFG; contract Si 609/16-1).

\section{Notes and references}

1 F. Hofmeister, Arch. Exp. Pathol. Pharmakol., 1888, 24, 247. 2 K. D. Collins, Methods, 2004, 34, 300.

3 V. Merk, C. Rehbock, F. Becker, U. Hagemann, H. Nienhaus and S. Barcikowski, Langmuir, 2014, 30, 4213.

4 Y. Li, Y. Wang, G. Huang, X. Ma, K. Zhou and J. Gao, Angew. Chem., Int. Ed., 2014, 53(31), 8074.

5 M. Dishon, O. Zohar and U. Sivan, Langmuir, 2009, 25(5), 2831.

6 J. M. Peula-Garcia, J. L. Ortega-Vinuesa and D. BastosGonzalez, J. Phys. Chem. C, 2010, 114, 11133.

7 T. Oncsik, G. Trefalt, M. l. Borkovec and I. Szilagyi, Langmuir, 2015, 31, 3799.

8 F. Zhang, D. G. Dressen, M. W. A. Skoda, R. M. J. Jacobs, S. Zorn, R. A. Martin, C. M. Martin, G. F. Clark and F. Schreiber, Eur. Biophys. J., 2008, 37, 551.

9 B. Lukanov and A. Firozabaadi, Langmuir, 2014, 30(22), 6373. 10 J. Lützenkirchen, Langmuir, 2013, 29, 7723.

11 S. S. Lee, P. Fenter, K. L. Nagy and N. C. Sturchio, Langmuir, 2012, 28, 8637.

12 A. Salis and B. W. Ninham, Chem. Soc. Rev., 2014, 43, 7358. 13 E. Thormann, RSC Adv., 2012, 2, 8297.

14 Y. A. Diaz Fernandez, T. A. Gschneidtner, C. Wadell, L. H. Fornander, S. Lara Avila, C. Langhammer, F. Westerlund and K. Moth-Poulsen, Nanoscale, 2014, 6, 14605.

15 N. Babajani, C. Kaulen, M. Homberger, M. Mennicken, R. Waser, U. Simon and S. Karthäuser, J. Phys. Chem. C, 2014, 118(46), 20657.

16 S. Bourone, C. Kaulen, M. Homberger and U. Simon, Langmuir, 2016, 32(4), 954.
17 C. Kaulen, M. Homberger, S. Bourone, N. Babajani, S. Karthäuser, A. Besmehn and U. Simon, Langmuir, 2014, 30, 574 .

18 B. Luk'yanchuk, N. I. Zheludev, S. A. Maier, N. J. Halas, P. Nordlander, H. Giessen and C. T. Chong, Nat. Mater., 2010, 9, 707.

19 H. H. Nguyen, J. Park, S. Kang and M. Kim, Sensors, 2015, 15, 10481.

20 A. Kumar, S. Kim and J.-M. Nam, J. Am. Chem. Soc., 2016, 138, 14509.

21 R. J. Stover, E. Moaseri, S. P. Gourisankar, M. Iqbal, N. K. Rahbar, B. Changalvaie, T. M. Truskett and K. P. Johnston, Langmuir, 2016, 32, 1127.

22 M. Ma, J. Wang and X. Zheng, Microchim. Acta, 2011, 172, 155.

23 B. Stein, D. Zopes, M. Schmudde, R. Schneider, A. Mohsen, C. Goroncy, S. Mathur and C. Graf, Faraday Discuss., 2015, $181,85$.

24 R. D. Deegan, O. Bakajin, T. F. Dupont, G. Huber, S. R. Nagel and T. A. Witten, Nature, 1997, 389, 827.

25 K. P. Browne and B. A. Grzybowski, Langmuir, 2011, 27(4), 1246.

26 M. C. Leopold and E. F. Bowden, Langmuir, 2002, 18, 2239.

27 T. C. Preston, M. Nuruzzaman, N. D. Jones and S. Mittler, J. Phys. Chem. C, 2009, 113(32), 14236.

28 J. Ruff, J. Steitz, A. Buchkremer, M. Noyong, H. Hartmann, A. Besmehn and U. Simon, J. Mater. Chem. B, 2016, 4, 2828.

29 J. J. Storhoff, A. A. Lazarides, R. C. Mucic, C. A. Mirkin, R. L. Letsinger and G. C. Schatz, J. Am. Chem. Soc., 2000, 122, 4640.

30 N. Schwierz, D. Horinek and R. R. Netz, Langmuir, 2015, 31, 215.

31 N. Schwierz, D. Horinek and R. R. Netz, Langmuir, 2013, 29, 2602.

32 E. F. Aziz, N. Ottosson, S. Eisebitt, W. Eberhardt, B. JagodaCwiklik, R. Vacha, P. Jungwirth and B. Winter, J. Phys. Chem. $B, 2008,112,12567$.

33 W. Zhou, X. Gao, D. Liu and X. Chen, Chem. Rev., 2015, 115, 10575.

34 V. Maheshwari, J. Kane and R. F. Saraf, Adv. Mater., 2008, 20, 284. 